ORIGINAL

\title{
EL DISCURSO DE LOS PROFESIONALES DE ATENCIÓN PRIMARIA DE LA COMUNIDAD DE MADRID ACERCA DEL TRABAJO CON GRUPOS: SOBRE TÉCNICAS Y TÉCNICOS
}

\section{Juan Carlos Duro Martínez $(1,2)$}

(1) Agencia Laín Entralgo para la Formación, Investigación y Estudios Sanitarios. Consejería de Sanidad. Comunidad de Madrid.

(2) Departamento de Medicina Preventiva, Salud Pública e Historia de la Ciencia. Universidad Complutense de Madrid

\section{RESUMEN}

Fundamentos: La Educación para la salud con grupos es una actividad cada vez más importante en atención primaria. Con el metaobjetivo de contribuir a su desarrollo, esta investigación se plantea como objetivos específicos: saber qué se entiende por trabajo con grupos, y conocer quiénes lo hacen y cómo se llevan a cabo las prácticas grupales.

Métodos: Desde una perspectiva cualitativa-estructural se realizaron cuatro grupos de discusión y cuatro entrevistas abiertas. Los grupos realizados fueron: dos con médicos, especialistas en pediatría, en medicina familiar y comunitaria, uno con profesionales de enfermería y otro con profesionales de trabajo social. Las entrevistas abiertas: dos con médicos generales y dos con trabajadores sociales de las Áreas de Atención Primaria de la Comunidad de Madrid.

Resultados: Los grupos se entienden, por la mayoría de los profesionales, como agrupación de individuos homogéneos respecto a la edad y a la patología a los que se les transmite información mediante 'charlas' que son hechas principalmente por enfermeras. Minoritariamente se reivindica otra manera de entender y hacer el trabajo con grupos, basado más en el intercambio de experiencias y en el fomento de la dinámica grupal, enfoque liderado sobre todo por las trabajadoras sociales. La participación de los médicos es minoritaria, esporádica y siempre en conjunto con las otras profesiones.

Conclusiones: El trabajo con grupos en la educación para la salud en atención primaria se refiere mayoritariamente a 'charlas' realizadas por enfermeras y trabajadores sociales, por lo que se ve necesario fomentar las actividades en equipo en el trabajo grupal, mejorando la motivación y la formación de los profesionales e incrementando la evaluación de las experiencias grupales.

Palabras clave: Educación para la salud. Atención primaria de salud. Grupos de discusión. Investigación cualitativa.

\section{Correspondencia:}

Juan Carlos Duro Martínez

C/ General Oráa, 3928006 Madrid

Correo electrónico: juancarlos.duro@madrid.org

\section{ABSTRACT}

The Discourse of the Primary Care

Professionals in the Autonomous

Community of Madrid Concerning the Work with Groups: on Techniques and Technicians

Background: Group-focused education for health is becoming a progressively more important activity in primary care. With the metaobjective of contributing to the further development thereof, ent is doing this work and how the group practices are carried out.

Methods: From a qualitative-structural standpoint, four discussion groups and four open interviews were held. The discussion groups held were: two with physicians, specialists in pediatrics, in family medicine and in community medicine, one with nursing professionals and another with professional social workers. The open interviews: two with general practitioners and two with social workers in the Primary Care Areas in the Autonomous Community of Madrid.

Results: The groups are understood, by most of the professionals, as groupings of homogeneous individuals as regards age and the disease involved to which information is conveyed by means of the disease involved to which information is conveyed by means of informal "talks" which are given mainly by nurses. A minority called
for another way of construing and doing work with groups based for another way of construing and doing work with groups based
more on a sharing of experiences and on fostering the group dynamics, an approach spearheaded particularly by women social workers. The physicians play a minority, sporadic role which is always in conjunction with the other professions.

Conclusions: Groups-focused work in education for health in primary care involves mostly "talks" given by nurses and social workers, it therefore being found necessary to promote team activiworkers, it therefore being found necessary to promote team activi-
ties in this group-focused work, by boosting the motivation and the training of the professional and bettering the evaluation of group experiences.

Key words: Focus groups. Health Education. Primary Health Care. Qualitative research. 


\section{INTRODUCCIÓN}

Este original está elaborado a partir de una investigación financiada por el Fondo de Investigaciones Sanitarias (Exp.93/0907) y sus resultados complementan y amplían los presentados en el artículo «El discurso de los profesionales de atención primaria de la Comunidad de Madrid sobre el trabajo con grupos: sentido, finalidades y ámbitos de intervención» publicado en esta misma Revista $^{1}$

Desde los iniciales planteamientos de las reformas sanitarias a nivel internacional, ya fueran referidas a la atención primaria ${ }^{2}$ o la promoción de la salud ${ }^{3}$, hasta las actuales directrices de la $\mathrm{OMS}^{4}$ pasando por las tendencias de planificación, organización y gestión de las instituciones sanitarias ${ }^{5-7}$, la prevención de la enfermedad y la promoción de la salud han formado parte constituyente, al menos en cuanto a los discursos se refiere, de una concepción de atención integral a la salud de las personas, familias y comunidades, siendo la educación para la salud un instrumento imprescindible para alcanzar objetivos preventivos y de promoción de la salud.

La educación para la salud, sin embargo, ha sido el 'patito feo' de la atención primaria $^{8}$, ha sido (y así continúa siendo) una actividad poco sistematizada que se hace 'si queda tiempo' después de la consulta ${ }^{1}$. El trabajo con grupos en el campo de la educación para salud ha tenido un desigual desarrollo en los últimos años. En instituciones sanitarias 'alternativas' durante la transición democrática y durante la primera época de la Reforma Sanitaria el trabajo con grupos irrumpió con fuerza en la, por entonces denominada, salud comunitaria (Centros de Promoción de la Salud de los Ayuntamientos, los Centros de Salud del Insalud, los Servicios de Salud Mental Comunitaria de la Comunidad de Madrid), acorde con los nuevos planteamientos de los enfoques biopsicosociales, de participación comunitaria y, sobretodo, de trabajo en equipo interdisciplinar'. La influencia de profesionales 'sociales' (psicólogos y trabajadores sociales fundamentalmente) en el discurso biomédico y en las prácticas sanitarias propició un acercamiento a 'lo grupal' por parte de algunos de los más inquietos médicos participantes en estos procesos de cambio ${ }^{10}$.

Paulatinamente el 'furor por los grupos' se fue apagando, al igual que los planteamientos comunitarios en pro de las Normas Técnicas Mínimas y de las Carteras de Servicios a partir de la denominada 'reforma de la Reforma' potenciada por el Informe Abril. Los restos del incipiente trabajo grupal se fueron esparciendo por equipos poco motivados para el trabajo 'más allá de la consulta' y se 'tecnificaron' con la inclusión formal del trabajo de grupos con crónicos, hipertensos y otras patologías en las Carteras de Servicios del Insalud que tuvieron un desigual grado de aceptación por parte de los profesionales ${ }^{11}$. Se produjo así el paso de entender el trabajo con grupos como un enfoque coherente con una forma de abordar los problemas de salud/enfermedad a ser entendidos como una cierta modalidad técnica, en el mejor de los casos, más eficiente que la atención individual para algunos de los problemas más frecuentes que se dan en atención primaria $^{12,13}$.

En cualquier caso, las intervenciones asistenciales individuales que se realizan en atención primaria, sobre todo por parte de los profesionales de la medicina y la enfermería, suelen tener un cierto componente 'educativo' al incluir, ya sea de manera bastante informal, consejos y recomendaciones preventivas y de promoción de salud o de manera protocolizada mediante el Programa de Actividades de Prevención y Promoción de la Salud (PAPPS) auspiciado por la Sociedad Española de Medicina Familiar y Comunitaria (SEMFyC). Esta práctica individual es más funcional al Modelo Médico Hegemónico ${ }^{14,15}$ en tanto remite los problemas de salud exclusivamente a las caracte- 
rísticas biológicas o comportamentales de cada sujeto obviando su carácter histórico y descontextulizando dichos problemas. Se refuerza así un modelo de dependencia profesional-usuario sin cuestionar el papel de ninguno de los dos. La educación para la salud grupal puede introducir elementos más novedosos en esa relación ${ }^{16}$, y en el mismo funcionamiento del equipo y la institución, al 'exigir' nuevas conceptualizaciones y elementos técnicos (encuadres) que cuestionen el discurso sanitario dominante ${ }^{17}$. No es raro, por lo tanto que su incorporación en la atención primaria haya sido mucho más lenta, irregular y sin el apoyo añadido que requiere por parte de la administración de manera que se ha limitado a recoger en la Cartera de Servicios 'charlas' en grupo con pacientes hipertensos y diabéticos fundamentalmente ${ }^{18}$.

Sin embargo las demandas en atención primaria, más allá de la definición de los pacientes como crónicos (en función del tiempo de la enfermedad), viejos (en función de la edad cronológica), mujeres (en función del género), etc. están cada vez más relacionadas con los determinantes de la salud como son el medio ambiente social y los modos y estilos de vida de las personas por lo que se requerirán planteamientos teóricos y técnicos que incidan en dichos determinantes de salud $^{19}$. Así en los planteamientos de la nueva Salud Pública será necesario tanto potenciar el control de las poblaciones sobre los determinantes sociales de su salud ('empoderamiento' $)^{20}$ como implementar encuadres de trabajo grupal que ayuden al cambio de valores, actitudes, creencias y comportamientos tanto de los 'profanos' (usuarios) como de los científicos (profesionales) en todo lo relativo al proceso salud/enferme$\mathrm{dad} /$ atención.

En este proceso es imprescindible contribuir todavía más el acercamiento de los conocimientos que provienen de las ciencias sociales (sociología, antropología, psicología social, etc.) al campo de la salud en gene- ral y de la atención primaria en particular adaptando al contexto histórico-social-institucional las experiencias que en el campo de lo grupal se han desarrollado en los últimos años, ya haya sido alrededor de la salud mental ${ }^{21}$, de la formación grupal de profesionales ${ }^{22,23}$ o explícitamente de educación para las salud ${ }^{24,25}$.

Con el metaobjetivo de propiciar un enfoque más psicosocial en el trabajo de promoción y educación para la salud en atención primaria, y partiendo de que también los propios profesionales son los que tienen que cambiar sus 'modos de entender y hacer', nos interesó conocer las distintas opiniones y valoraciones sobre las concepciones, modalidades y técnicas de trabajo con grupos en educación para la salud en atención primaria, es decir, conocer los discursos de dichos profesionales sanitarios sobre qué entienden por grupos, cómo se llevan a cabo esas prácticas grupales y quiénes las realizan. Esos fueron los objetivos específicos de la investigación que vamos a exponer en este artículo.

\section{SUJETOS Y MÉTODO}

Teniendo en cuenta los objetivos de investigación propuestos, entre los diferentes métodos cualitativos se optó por el método estructural que toma como objeto de investigación a los sujetos-actores para tratar de captar sus opiniones y experiencias de las personas desde su mundo simbólico ${ }^{26,27}$. Como técnicas de captura de datos se utilizaron grupos de discusión y como complemento las entrevistas abiertas ${ }^{28,29}$ para saturar el discurso grupal de los trabajadores sociales y completar el discurso médico con médicos generales sin la especialidad de Medicina Familiar y Comunitaria.

Podemos por lo tanto dividir a la población objeto de estudio en función de diferentes variables relevantes para nuestros objetivos de investigación: profesión, área sanita- 
Tabla 1

Diseño de los Grupos de discusión y las entrevistas abiertas

\begin{tabular}{|c|c|c|c|}
\hline & Profesionales & Área Sanitaria & Sexo/Edad \\
\hline Grupo de Discusión l & 9 profesionales de enfermeria & Areas $1,2,5,9,10$ y 11 & $\begin{array}{l}8 \text { mujeres y } 1 \\
\text { hombre } \\
30-40 \text { aถ̃os }\end{array}$ \\
\hline Grupo de Discusión 2 & $\begin{array}{l}7 \text { medicos especialistas en } \\
\text { Medicina de Familia y } \\
\text { Comunidad y Podiatria }\end{array}$ & Áreas $1,3,6,8,10$ y 11 & $\begin{array}{l}4 \text { mujeres y } 3 \\
\text { hombres } \\
30-45 \text { affos }\end{array}$ \\
\hline Grupo de Drscusión 3 & 5 trabajadoras sociales & Árens $1,8,9$ y 10 & $\begin{array}{l}5 \text { mujeres } \\
26-35 \text { an̂os }\end{array}$ \\
\hline Grupo de Drscusión 4 & $\begin{array}{l}6 \text { médicos de Medicina } \\
\text { General y especialistas en } \\
\text { Medicina de Familia y } \\
\text { Comunidad }\end{array}$ & Árens $4,7,8,9$ y 11 & $\begin{array}{l}5 \text { mujeres y } 1 \\
\text { hombre } \\
30-40 \text { años }\end{array}$ \\
\hline 2 Entrevistas en profundidad & Médicos generales & Areas 3 y 6 & $\begin{array}{l}\text { hombres } \\
35-40 \text { años }\end{array}$ \\
\hline 2 Entrevistas en profundidad & trabajadoras sociales & Arens 2 y 5 & $\begin{array}{l}\text { mujeres } \\
30 \text { anhos }\end{array}$ \\
\hline
\end{tabular}

Tabla 2

Variables para la composición de los Grupos de Discusión

- Colectivo profesional de pertenencia: médicos generales y médicos especialistas en pediatría, médicos especialistas en medicina familiar y comunitaria, enfermeros y trabajadores sociales.

- Área Sanitaria en la que se desempeña el trabajo

- Hombres y mujeres

- Un mínimo de dos años de experiencia en Atención Primaria

- Formación/experiencia en trabajo con grupos

ria y género. El resto de variables hay que tenerlas en cuenta para buscar la mayor heterogeneidad inclusiva en el discurso de cada clase ${ }^{30}$. Son especialmente relevantes: el tiempo de actividad laboral y, sobre todo, si han tenido formación y/o experiencia de trabajo grupal y/o psicosocial.

Así se organizaron los grupos (tablas 1 y 2) en función de la variable profesión. La variable Área sanitaria se tuvo en cuenta para evitar la excesiva representación de aquellas áreas en las que hubiera un mayor desarrollo de las actividades grupales. La variable experiencia previa con el trabajo con grupos, aunque fue tenida en cuenta a la hora de la composición de los grupos, no ha podido ser identificada cuantitativamente en cada grupo por no haber llevado los investigadores un registro de la misma ya que fueron los captadores los que la tuvieron en cuenta a la hora de seleccionar a los participantes en los grupos de discusión. Esta limitación hace que, aunque los resultados expresan la tendencia del posicionamiento topológico de las opiniones en función de los

Rev Esp Salud Pública 2003, Vol. 77, N. ${ }^{\circ} 5$ 
distintos niveles de experiencia, éstos no se puedan relacionar con claridad con la experiencia grupal previa de los participantes. El proceso de captación empleado ha sido el método de 'bola de nieve' o 'red de captación mediante pasos sucesivos'.

Los cuatro grupos de discusión y las cuatro entrevistas abiertas que forman parte de esta investigación se realizaron en el Centro Universitario de Salud Pública, institución docente vinculada a la Universidad Autónoma de Madrid y a la Comunidad de Madrid y cuya imagen de neutralidad respecto a la institución Insalud a la que pertenecen todos los participantes es condición metodológica necesaria para la fluidez del habla de los participantes. La duración de cada reunión de grupo osciló entre los setenta y cinco y los noventa minutos. En todos los grupos y entrevistas se invitó a la discusión mediante la consigna: «vamos a hablar del trabajo cotidiano que se hace fuera de la consulta en los equipos», para después, según la guía temática (tabla 3), ir centrando el debate en las actividades o trabajos con grupos, una vez que se nombraba de forma espontánea por los hablantes.

Todo lo dicho en los grupos de discusión y entrevistas abiertas fue recogido en cinta magnetofónica y posteriormente transcrito de forma literal. El análisis y la interpretación de la información recogida se realizaron mediante la organización del material respecto a los objetivos, la definición de epígrafes y la redacción significativa a partir de los conocimientos y experiencias de los investigadores. El análisis fue realizado, en primera instancia, por el investigador/preceptor de los grupos y posteriormente fue contrastado y supervisado por el investigador principal del estudio. Ninguno de los dos investigadores conocía ni tuvo contacto previo con los integrantes de los grupos y las entrevistas abiertas como recomienda el enfoque cualitativo-estructural utilizado en este estudio ${ }^{31}$.

\section{RESULTADOS}

Bajo la denominación de trabajo con grupos se engloban diferentes concepciones teóricas, metodológicas y técnicas que se pueden desbrozar a partir del análisis de lo que podemos denominar 'el discurso del cómo'. Se incluye en dicho discurso dos aspectos acerca de qué concepción se tiene de los grupos, qué técnicas grupales se utilizan (y/o deberían utilizarse) y sobre quién y cómo -qué técnicos- los hacen (y/o deberían hacerlos). La organización en epígrafes facilita la exposición de los resultados que se acompañan de los dichos (verbatim) más relevantes (tabla 4).

\section{Concepciones grupales y modalidades técnicas}

Las concepciones que se tienen sobre los grupos determinan el uso de las técnicas grupales. Evidentemente en el ámbito sanitario en general y de la atención primaria en particular el debate sobre estas cuestiones está lejano por lo que se impone en la práctica una visión del grupo más desde el sentido

Tabla 3

Guía temática
- Valoración sobre actividades grupales hechas por los profesionales de APS.
- ¿Qué profesionales participan más en este tipo de actividades?
- Técnicas grupales utilizadas.
- Actividades grupales y trabajo en equipo. 


\section{Verbatim más relevantes}

$\mathrm{N}^{\circ} 1$ «...son charlas a un grupo de 15 personas, ¿entiendes?, o sea que la diferencia es importante, y nosotros sí que les dimos 3 charlas, una de alimentación, otra de sexualidad, y otra a nivel psicológico, y se les dieron esas tres, pero, vamos, que era un grupo de 15 personas, cuando se acabaron las tres se fueron, vino otro y se hizo con 30 mujeres...» E TS.

$\mathrm{N}^{\circ} 2$ «O sea, es que ahora te mandan dirigir grupos, tú dices pues muy bien, pues les echo una charla y que salga lo que Dios quiera, pues claro que puede salir lo que quiera...» GDM.

$\mathrm{N}^{\mathrm{o}} 3$ «Y yo la experiencia que he visto es que no son grupos, no se hacen grupos, se hace información que en vez de darse de forma individualizada se da forma colectiva [...] yo creo que es ahí donde nosotros podemos más dinamizar, asesorar» GDTS.

$\mathrm{N}^{\circ} 4$ «Porque el grupo no es solamente llegar y dar una información sobre lo que es una enfermedad o un problema de salud [...] yo creo que la fuerza del grupo está en que ahí hay una gente que tiene una fuerza estupenda y que se pueden reforzar unos a otros, convencer, fundamentárselo mejor que tú, en su idioma, en su lenguaje, en su preocupación, en sus angustias, o sea que es una forma de entender el problema, no solamente que tú le manifiestes esa historia» GDE.

$\mathrm{N}^{\circ} 5$ "Es importante, porque el grupo es una forma de aprendizaje también, ¿no?, es una forma de romper cosas, es una forma de ayuda mutua entre las personas que tienen un problema y eso es terapéutico, y eso es enriquecedor y eso mejora la calidad de vida... Y al mismo tiempo mejora la asistencia porque puede haber menos gente, o todos esos casos que... repetitivos en consulta, que no vienen por nada en concreto sino por sus angustias, porque son hiperutilizadores de los servicios médicos; pues bueno puedes canalizar esas angustias o esos problemas» GDTS.

$\mathrm{N}^{\circ} 6$ «Estamos pidiendo que sea una prescripción sanitaria... son derivados para mí. O sea que una prescripción facultativa o sanitaria va a ser acudir a ese grupo que está ahí como recurso del centro» GDTS.

$\mathrm{N}^{\mathrm{o}} 7$ «En mi centro partió antes de que entrara en Cartera de Servicios como inquietud de algunas enfermeras» $E M G$.

$\mathrm{N}^{\circ} 8$ «Yo he estado en grupos de diabetes, y ahí lo que tú dices, es superenriquecedor, es que sales tú aprendiendo. Yo he estado en un grupo de diabéticos y he acabado sabiendo cosas de diabetes que no sabía, ¿sabes?, porque empiezan a contarse unos a otros, empiezan a preguntar dudas y entre ellos se las van solucionando» GDM.

$\mathrm{N}^{\circ} 9$ «Y eso lo hace más enfermería, porque tiene más tiempo para esas cosas. Enfermería y el equipo técnico de primaria quizá tengan más tiempo para hacer cosas de ésas, y el que más hincapié tiene que poner en eso... Pero es que enfermería tiene un problema que ha sido saber qué papel tiene en Atención Primaria» GDM.

$\mathrm{N}^{\circ} 10$ «Yo estoy de acuerdo, en que no saben todavía cuáles son sus funciones y su área de trabajo .... por eso mismo ahora se dedican a cosas de ninguna manera contrastadas, y ¿por qué?, para llenar su tiempo, ¿por qué?, porque no tienen efectivamente un marco de actividades con unos contenidos ....-. Yo lo que quiero es una enfermera-enfermera, bien claro, si luego además tiene tiempo para lo otro, fenomenal, pero claro, que sólo la dediquen a lo otro, me opongo, [...] que a mí la charla me parece muy bien siempre que lo otro esté cubierto» EMG.

$\mathrm{N}^{\mathrm{o}} 11$ «...nosotros estamos padeciendo ..... - antes teníamos asistente social y ahora tenemos charlistas, entonces es triste, yo es que no quiero un charlista más ....- como favor especial te tramita lo de la silla de ruedas de tus viejos pero no por trabajo, porque ella ahora su trabajo es dar charlas a señoras de 40 años ....-, pero, claro, para mí es fundamental que el viejo tenga su silla de ruedas»E $M G$.

$\mathrm{N}^{\mathrm{o}} 12$ «...pues lo primero que habría es que definir qué es lo que tendríamos que hacer, pero quizá el camino es ése, ¿no?, la educación a grupos, el hacer mucho más [atención] domiciliaria, el hacer otro tipo de actividades que no es la consulta de enfermería ....- siempre las enfermeras tenemos que ganarnos nuestras cosas como Juana de Arco» GDE.

EMG: Entrevista a Médico General. ETS: Entrevista a Trabajadora Social. GDM: Grupos de Discusión de Médicos. GDTS: Grupo de Discusión de Trabajadores Sociales. GDE: Grupo de Discusión de enfermería. 
común (suma de individuos) que desde cualquier teoría propia de la psicología social y grupal (el grupo como instancia distinta a la suma de individuos). Coherentemente con esta concepción del grupo como 'agrupamiento' de personas la técnica privilegiada será la 'charla' en grupo en la que los participantes serán un auditorio, colectivo más bien pasivo, antes que constructores activos de su propia dinámica grupal.

\section{«Pues les echo una charla y que salga lo que Dios quiera»}

Parece haber un alto grado de consenso en que la técnica dominante de hecho para el trabajo con grupos de pacientes (y especialmente) con patologías crónicas es la "charla'. Ésta se describe como un escenario en el que se reúnen a pacientes con la misma patología y relativamente homogéneos en otras variables (sobre todo, edad y nivel cultural) a los que se les informa colectivamente de contenidos relativos a su enfermedad o situación y consejos para sobrellevarla (cómo identificar síntomas, qué hacer en distintas situaciones, cómo alimentarse, qué autocuidados y tratamientos son útiles para cada caso, etc.). También se dice que suele ser un número pequeño de 'charlas' o sesiones, lo que hace que sean intervenciones más bien cortas y sin continuidad en el tiempo. (verbatim 1)

A tenor de las diferencias encontradas en el discurso entre los profesionales que tenían experiencia en el trabajo con grupos y los que no tenían, cabe decir que la experiencia (más que la formación teórica o antes que ésta) se constituye como foco de aprendizaje para el profesional y recrea un espacio en el que construir otra idea de lo que es el grupo, más allá de la charla. Es decir, el contacto directo con un grupo (tanto si se es el encargado del mismo como si se es observador) permite incluir elementos en la definición -y experiencia subjetiva- del grupo, que tienen que ver fundamentalmente con el ámbito relacional/participativo (entre los miembros del grupo y entre éstos y el técnico) que no están presentes en la definición del grupo como "charla'.

Los profesionales con cierta experiencia en trabajo con grupos apuntan ciertos requisitos técnicos como necesarios para el desarrollo correcto de las 'charlas': fundamentalmente, consisten en adecuar/adaptar el lenguaje médico-científico al nivel cultural de los participantes en los grupos, y planificar un tiempo de debate tras la exposición de contenidos para que surjan las dudas posibles y aclararlas por parte del profesional, además de permitir la puesta en común de inquietudes, necesidades y vivencias de los pacientes -lo que, desde el punto de vista de algunos profesionales, enriquece el resultado del trabajo con grupos frente al individual-.

El hecho de que la 'charla' sea en el discurso, la técnica más re-conocida (en el sentido de que se conoce que se utiliza) no significa que la valoración de dicha técnica sea homogénea entre los profesionales. De hecho, es posible detectar posturas diferentes. Precisamente, la cita elegida como epígrafe de este apartado pone de manifiesto una postura crítico-reflexiva enarbolada por algunos profesionales, entre los que se incluyen algunos médicos, pero sobre todo integrantes de los otros dos colectivos, enfermería y trabajo social. Dicha postura crítica parte de la base de la distinción esencial entre el trabajo clínico y el educativo, y de la toma de conciencia de la falta de preparación o formación para llevar a cabo actividades del ámbito educativo (verbatim 2).

Esta demanda de formación tiene que ver con la percepción del grupo como un espacio abierto (con más público que puede comentar y juzgar lo que el profesional dice), y más 'amenazante', frente a la consulta individual como espacio cerrado y oculto a la vista de los demás. 


\section{«...es que no son grupos, se hace información colectiva»}

Dentro del personal de enfermería y, sobre todo, de trabajo social se formaliza una crítica más elaborada a la 'charla': consideran que esta técnica no es adecuada a los fines y objetivos propuestos, así como que no cumple la propia definición de grupo (contemplada desde su formación de pregrado) (verbatim 3).

En este sentido, incluso aunque lo hagan ellos, contemplan este modelo como el 'médico-informativo' y sostienen que para otras técnicas o abordajes no hay lugar en atención primaria, bien porque la organización no está preparada para enfoques distintos, porque otro tipo de grupos no se percibiría como 'rentable' (en cuanto a tiempo y número de profesionales dedicados, etc.) o porque los profesionales con más autoridad -médicos- expresarían su escepticismo y resistencia a enfoques menos directivos. Ingredientes de estos otros enfoques serían, según algunos enfermeros/as y trabajadores/as sociales: la participación más activa de los miembros del grupo, la consideración de la dinámica grupal y el carácter único y distinto de cada grupo, la continuidad en el tiempo (o búsqueda de resultados a medio-largo plazo) y la implicación del profesional en el proceso. (Verbatim 4). En este sentido, el carácter terapéutico (con efectos en la calidad de vida, en sentido general) se centra en una definición del grupo como un escenario o dispositivo de escucha y de contención de 'angustias'. La escucha y elaboración de estas 'angustias' proporcionaría un bienestar y una mejora terapéutica. Según estos profesionales, el dispositivo grupal es de los pocos, en atención primaria, que cumplen esta función (verbatim 5).

Minoritariamente, algunos profesionales de enfermería y trabajo social con formación extra en su ámbito privado, incorporan la técnica de grupo operativo como otra técnica posible a utilizar; sin embargo, ellos mismos apuntan la falta de rentabilidad de dicho enfoque, desde el momento en que hacen falta dos o tres profesionales (coordinador, observador/es) durante un período largo de tiempo, con resultados quizá no tangibles.

Los grupos de educación para la salud en la escuela (con maestros) parecen tener un carácter más formativo, pero también con la 'charla' como técnica, en el sentido de transmitir a los maestros un contenido médico-científico que, después, ellos transformarán en contenidos más adecuados para los niños mediante su conocimiento de la didáctica (entendido como arte de transmitir/enseñar a otros).

\section{«Que una prescripción sea acudir a ese grupo...»}

Con este epígrafe nos referimos al deseo expresado por algunos trabajadores sociales de que la captación de los participantes de un grupo (o, en última instancia, la organización del grupo) sea una labor de equipo y no sólo de los profesionales individuales que vayan a realizar la actividad. Si se tratara de una prescripción facultativa (como otras posibles: medicamentos, dietas, etc.) significaría que el equipo ha consensuado y aceptado la intervención como un proyecto de equipo, con unos criterios de selección comunes así como los objetivos. Este trabajo en equipo supondría también la incorporación del colectivo de trabajo social al equipo y no sólo su aportación como personal de apoyo (verbatim 6).

Sin embargo, la expresión de este deseo de forma explícita se hace porque su realidad observada no es ésa: se dice que la captación o la organización de los grupos suele correr a cargo de las personas que llevan la iniciativa del trabajo grupal, bien recurriendo a las historias clínicas, bien derivando desde las propias consultas, pero pocas veces como un trabajo consensuado de equipo. 


\section{¿Quién hace y quién debería hacer los grupos?}

Es éste uno de los puntos de fricción más importantes entre los distintos colectivos profesionales, con consecuencias en otros terrenos conexos, como el establecimiento y definición de las competencias de cada colectivo (en última instancia, la identidad profesional) y del equipo, y el reconocimiento y valoración del trabajo de los otros por parte de cada colectivo. En otras palabras, la consideración de quién debería hacer el trabajo con grupos está atravesada por la actitud hacia dicha actividad y por el significado y valoración atribuidos a cada colectivo dentro del marco de la atención primaria.

\section{«La educación para la salud grupal la hace quien está motivado, suelen ser enfermeras y trabajadoras sociales»}

Haciendo historia de los años más recientes, los profesionales consultados apuntan a que el trabajo con grupos ha sido una actividad minoritaria llevada a cabo, fundamentalmente, por personas motivadas para ello, a título más bien individual (verbatim 7). De hecho, está implícita la necesidad de cierto grado de voluntariedad en la realización de estas actividades frente a la obligatoriedad o imposición de las mismas. Hay acuerdo entre los tres colectivos en que estas iniciativas individuales son más patrimonio de enfermería y trabajo social.

Los profesionales mencionan la experiencia directa o el contacto con el trabajo con grupos como una fuente de efectos o consecuencias para ellos. Uno de ellos es la ruptura de la rutina o la monotonía cotidiana, al realizar una actividad fuera de lo habitual, definida -entre otros elementos- por la relación social que supone. Además, aporta para ellos un conocimiento de los pacientes desde otra perspectiva distinta a la que ofrece la consulta individual, junto con un enfoque también distinto de la enfermedad de estos pacientes (la enfermedad desde el mundo y visión de los pacientes). Algunos profesionales engloban estos procesos dentro de otro más amplio: un enriquecimiento personal, mediado por el aprendizaje desde la experiencia (verbatim 8 ).

\section{«Lo hace más enfermería, porque tiene más tiempo para esas cosas»}

En lo que hay más diversidad es en las explicaciones o interpretaciones que cada colectivo da a este aparente reparto de trabajo. Una de las explicaciones dominantes entre el personal médico es que el trabajo con grupos recae sobre las enfermeras porque éstas disponen de más tiempo durante su jornada laboral. El hecho de disponer de más tiempo responde, según aquéllos, a un relativo vacío de funciones o competencias del personal de enfermería que se percibe como una situación de agravio comparativo entre ambos colectivos en relación a dos aspectos: en cuanto a la carga de trabajo y en cuanto al sentido y utilidad del trabajo dentro del marco de la atención primaria (verbatim 9).

La radicalización de esta última postura unida a una actitud escéptica hacia el trabajo con grupos lleva, entre algunos médicos, a la descalificación de las actividades de enfermería: perciben que el resultado de dichas actividades no repercute en el trabajo médico-clínico, añorando, por el contrario, la figura de enfermería vigente en el modelo asistencial anterior a la reforma sanitaria (una 'enfermera-enfermera'). La actitud escéptica o descalificadora del trabajo con grupos $-y$, en última instancia, del trabajo de enfermería- tiene que ver con la consideración central que, para ellos, tiene la práctica clínica (que se manifiesta en una reivindicación de mayores competencias clínicas) y la consideración periférica de 'lo otro' (la educación para la salud, el trabajo con grupos, etc.) (verbatim 10).

Esta postura crítica se extiende también hacia los trabajadores sociales que, desde 
este punto de vista, abandonan sus obligaciones -que repercuten y sirven de apoyo técnico al espacio clínico- para dedicarse a 'lo otro' (mereciendo una descalificación se les llama 'charlistas'- o, al menos, un no reconocimiento de este 'otro' trabajo). En este sentido, se pondrían de manifiesto dos de los problemas centrales que dispara el tema del trabajo con grupos: la falta de consenso y debate interno sobre sus significados y sentidos, y la división de los equipos de atención primaria entre el personal médico y los otros colectivos, que vuelve a hablar de ausencia de proyectos de centro o trabajo en equipo (verbatim 11).

En mayor o menor grado, el personal de enfermería y trabajo social reconoce la existencia de la actitud del colectivo médico descrita más arriba, que se percibe como un gradiente que va desde la no colaboración (dentro del laissez-faire) hasta la falta de apoyo u oposición. Entre los trabajadores sociales esta percepción hace surgir tres posturas que tienen que ver con la búsqueda y defensa de su identidad profesional: o bien llevar adelante sus propios grupos, integrados por los usuarios de su consulta (sobre todo, grupos de mujeres), para los que dicen no necesitar a los profesionales sanitarios o bien hacer una 'subterránea' labor de formación hacia otros miembros del equipo, labor que, en ocasiones, les ha hecho merecedores del calificativo de 'insistentes sociales' (porque se dice que, por ejemplo, están constantemente recordando los determinantes psicosociales de la salud, etc.), o bien, evitar el 'voluntarismo' o 'individualismo' con el argumento de que estas actividades deben ser trabajo del equipo (y no sólo de trabajo social) o, en caso contrario, 'carecen de sentido'.

Por su parte, el colectivo de enfermería oscila entre la reivindicación del escenario del trabajo con grupos en solitario (es decir, sólo para el colectivo) para ir consolidando la identidad profesional como colectivo, y, en particular, también con pacientes sanos, a la consideración de que el trabajo con grupos debe ser objetivo del equipo y no sólo del colectivo de enfermería (en la línea integradora de los trabajadores sociales) (verbatim 12).

A modo de resumen destacaremos que la 'charla' resulta ser la técnica de trabajo grupal más mencionada y que ello apunta al modelo médico-informativo de educación para la salud (como modelo dominante aunque no único). En el extremo, la transmisión de información y de contenidos supone un enfoque vertical de la educación (de arriba hacia abajo), manifestado también en el reparto de 'papeles' entre los profesionales a la hora de participar en las actividades grupales: así, los profesionales sanitarios se ocuparían de los contenidos clínico-sanitarios (dentro de su área de conocimientos) mientras que los trabajadores sociales se encargarían de la dinamización, coordinación y captación. En este sentido, el trabajo con los grupos no acabaría de constituir un objetivo del equipo como tal (enmarcado en un proyecto colectivo), sino más bien actividades individuales de profesionales que, eventualmente, piden la colaboración de otros profesionales para cubrir 'lagunas' de contenido que ellos no puedan impartir.

Las dificultades para la realización de grupos, según los profesionales, son: la falta de formación de pregrado y continuada, su no inclusión como objetivos y actividades de los equipos, el 'miedo escénico' a la ‘exposición' pública y una organización del trabajo que no facilita (en tiempos de dedicación y recursos) las actividades grupales, en la que se incluye el poco trabajo en equipo y la falta de apoyo por parte de los coordinadores de los equipos y las gerencias de Área.

Las demandas de mejora se refieren por lo tanto al aumento de apoyo y reconocimiento institucional, la potenciación de investigaciones sobre las experiencias, ampliar y diversificar el tipo de grupos, dotar de recursos físicos y desarrollar colaboraciones con 
otras instituciones de sus zonas para darle un sentido comunitario a las intervenciones grupales.

\section{DISCUSIÓN}

El discurso mayoritario de los profesionales de atención primaria sobre los grupos está teñido del individualismo que sostiene el Modelo Médico Hegemónico, es decir los grupos como yuxtaposición, como suma de elementos individuales que permiten un trabajo de 'educación/información' 'como si' estuvieran formados por una persona 'clonada' muchas veces. Los grupos no merecerían un nivel de análisis diferenciado de 'lo individual', de orden cualitativamente diferente (estructura y dinámica grupal) sino que se quedan reducidos a un sumatorio de elementos en fase de serialidad.

Igualmente el trabajo grupal no plantearía mayores requerimientos técnicos que 'dar bien una charla' a un auditorio más o menos restringido sobre temas 'médicos'. Es un bonito emergente de esta concepción de los grupos el verbatim «pues les echo una charla y que salga lo que Dios quiera». Los participantes como receptores pasivos a los que el técnico les vierte 'sabiduría' según lo que Freire llamaba concepción bancaria de la educación $^{32}$.

El hecho de concebir el trabajo grupal como 'charlas', sin que por ello exijan una mayor cualificación tecnológica para quienes las imparten, permite que sea el personal de enfermería y/o de trabajo social (en posición subalterna al personal médico), quienes principalmente se hagan cargo de las "charlas' ya con el beneplácito de los médicos, ya con su ácida oposición o, en la mayoría de los casos con una silente indiferencia. Esta ‘adjudicación' del rol de 'charlistas' o 'insistentes sociales' no es ajena al proceso de reivindicación/asunción de mayor protagonismo en la educación para la salud por parte de enfermería (y en menor medida de los/las trabajadores/as sociales) $)^{33,34}$. Este interés por parte de enfermería, trabajo social y algunos médicos, conscientes de la insuficiencia de este planteamiento, ha hecho que en algunas Comunidades Autónomas se haya sistematizado el trabajo grupal en atención primaria con el asesoramiento y en apoyo de salud pública, como es el caso de Navarra en el que se intenta fomentar una educación para la salud grupal que combine el aprendizaje de conocimientos con el cambio de actitudes y el desarrollo de habilida$\operatorname{des}^{35,36}$. En Madrid, en buena medida propiciado por actividades de formación realizadas desde el Centro Universitario de Salud Pública en los años 90 y desde la misma institución sanitaria a través de la formación continuada potenciada por los responsables de Educación para la salud de las 11 Áreas sanitarias se está potenciando el trabajo de Educación para la salud grupal como lo muestra la publicación de unas «Recomendaciones metodológicas básicas para elaborar un proyecto educativo» ${ }^{37}$. Vemos pues que la reivindicación expresada en esta investigación por parte de aquellos profesionales con más experiencia en el trabajo grupal de fomentar 'otra forma' de hacer grupos en las que se fomente la implicación y participación de los pacientes intercambiando inquietudes y vivencias, en algunos casos, tiene algún eco por parte de las instituciones sanitarias, aunque todavía de manera poco generalizada.

La 'evaluación' realizada por los participantes en este estudio sobre los efectos de los grupos ya señalada en el artículo precedente $^{1}$ muestra el déficit de estudios evaluativos en nuestro contexto, más allá de evaluaciones sobre conocimientos adquiridos pone en evidencia la necesidad de evaluar distintas experiencias tanto desde una perspectiva cuantitativa (coste-eficacia, impacto en salud, etc. $)^{38,39}$ como cualitativa (motivaciones, satisfacción, etc.) en el campo de la Promoción y la Educación para la Salud $^{40-42}$. 
Por último la necesidad de ligar el trabajo con grupos con el trabajo en equipo y con la pertenencia a un proyecto colectivo propio de cada centro coincide con demandas expresadas en otros estudios sobre expectativas y organización de los centros por los profesionales de atención primaria ${ }^{43-44}$.

\section{AGRADECIMIENTOS}

A Celia Arroyo por su participación en la investigación, al Centro Universitario de Salud Pública por posibilitar su realización y a los profesionales que aportaron su discurso por permitir que nosotros lo deconstruyamos para construir el nuestro.

\section{BIBLIOGRAFÍA}

1. Duro Martínez JC. El discurso de los profesionales de atención primaria de la Comunidad de Madrid sobre el trabajo con grupos: sentido, finalidades y ámbitos de intervención. Rev Esp Salud Pública 2002;76: 545-559.

2. OMS. Informe de la Conferencia Internacional sobre Atención Primaria de Salud. Ginebra: OMS; 1978.

3. OMS. Carta de Otawa para la Promoción de la Salud.. Ginebra; OMS; 1986.

4. Ministerio de Sanidad y Consumo, OMS. Salud 21: Salud para todos en el siglo XXI. Madrid: Ministerio de Sanidad y Consumo; 1999.

5. Martín Zurro A, Ledesma Castelltort A, Sans Miret A. El modelo de atención primaria de salud: balance y perspectivas. Atención Primaria 2000;25: 100114

6. Repullo JR. La Atención Primaria ante los nuevos retos de gestión. Centro de Salud 2000;8: 18-25..

7. Arévalo C, Arnal R, Font M, Gómez T, Illan F, Jiménez C et al. La Atención Primaria. Administración Sanitaria 2001;5: 717-746.

8. Duro Martínez JC. Educación para la Salud: ¿quién la quiere?. $2^{\text {a }}$ Jornadas de Puesta en común en Atención Primaria. Sector VIII-Madrid. Madrid: Insalud; 1992.p. 87-92.
9. Duro Martínez JC. Psicología y Salud Comunitaria durante la Transición Democrática. Clínica y Salud 2001; 12: 1-175.

10. Duro Martínez JC. Discursos y prácticas profesionales de psicología social en salud comunitaria 1970-1995.-. Tesis Doctoral. Departamento de Sociología y Antropología Social. Universidad Autónoma de Madrid;1999.

11. Pérez Andrés C. Aceptación del Contrato Programa por el personal sanitario de la Atención Primaria de Madrid: un estudio cualitativo. Rev Sanid Hig Pública 1995; 69: 79-88.

12. Saez S, Marques F, Colell R. Técnicas de dinámica de grupo aplicables a la Educación para la salud. Educación para la salud. Lleida: Pagés Editors; 1995.p. 47-82.

13. Tormo Molina J, Rodríguez Fernández MJ, Hernán García M, Fernández Ajuria A. Educación para la salud en escuelas de adultos: ¿por medio del profesor o mediante charla de educación sanitaria? Aten Primaria 2000; 25: 242-247.

14. Menéndez E. El Modelo Médico Hegemónico: transacciones y alternativas hacia una fundamentación teórica del modelo de autoatención en salud. Arxiu d'Etnografía de Catalunya 1984; 3: 83-119..

15. Menéndez E. Morir de alcohol. Saber y hegemonía médica. México: Alianza Editorial Mexicana; 1990.

16. Sánchez Candamio M. Educación para la salud en grupo: acotaciones conceptuales y terminológicas sobre un proceso de cambio. Clínica y Salud 1994;5: 281-287.

17. Sánchez Moreno A, Ramos E, Sánchez V, Marset P. Estructuras de racionalidad en Educación para la Salud. Rev Sanid Hig Pública 1995; 69: 5-15.

18. Insalud. Cartera de Servicios en Atención Primaria. Definiciones, Criterios de Acreditación, Indicadores de Cobertura y Normas Técnicas Mínimas. Actualización en 2000, $4^{\mathrm{a}}$ edición 2001 ed. Madrid: Instituto Nacional de la Salud. Subdirección General de Coordinación Administrativa. Área de Estudios, Documentación y Coordinación Normativa; 1995 .

19. Colomer C, Álvarez-Dardet C. Promoción de la salud: concepto, estrategias y métodos. En: Colomer C, Álvarez-Dardet C, editores. Promoción de la Salud y Cambio Social. Barcelona: Masson; 2001.p. 27-44.

20. Ministerio de Sanidad y Consumo, OMS. Promoción de la salud. Glosario. Madrid: Ministerio de Sanidad y Consumo; 1998.

Rev Esp Salud Pública 2003, Vol. 77, N. ${ }^{\circ} 5$ 
21. Bauleo A, Duro JC, Vignale R. La Concepción Operativa de Grupo. Madrid: Asociación Española de Neuropsiquiatría; 1990.

22. Tizón JL. Los grupos de reflexión en atención primaria de salud II. Algunos elementos teóricos y técnicos. Atención Primaria 1993;11: 63-70..

23. Duro Martínez JC. La formación grupal de profesionales en instituciones públicas. Clínica y Salud 1992; 3: 77-84..

24. Pérez Jarauta MJ, Echauri Ozcoidi M. Instrumentos de la promoción de la salud. Dimensión Humana $1998 ; 2$ : 35-44.

25. Pérez Jarauta MJ, Echauri Ozcoidi M. Educar en Salud. Pamplona: Gobierno de Navarra; 1999.

26. Pérez Andrés C. Sobre la metodología cualitativa. Revista Española de Salud Pública 2002; 76:373380 .

27. Fernández de Sanmamed MJ. Introducción a la investigación cualitativa. Formación Médica Continuada en Atención Primaria 1995;2: 23-28..

28. Ortí A. La apertura y el enfoque cualitativo o estructural: La entrevista abierta semidirectiva y la discusión de grupo. En: Alvira F, García Ferrando M, Ibáñez J, editores. El análisis de la realidad social. Madrid: Alianza Universidad; 1989.p. 153185 .

29. Alonso LE. Sujeto y discurso: el lugar de la entrevista abierta en las prácticas de la sociología cualitativa. En: Delgado JM, Gutiérrez J, editores. Métodos y técnicas cualitativas de investigación en Ciencias Sociales. Madrid: Síntesis; 1994.p. 225240.

30. Buzzaqui A, Uris J. El grupo de discusión. Una herramienta para la investigación en Atención Primaria. Formación Médica Continuada en Atención Primaria 1997; 4:421-433.

31. Ibáñez J. Cómo se hace una investigación mediante grupos de discusión. En: García Ferrando M, Ibáñez J, Alvira F, editores. El análisis de la realidad social. Madrid: Alianza Editorial; 1990.p. 489-501.

32. Freire P. La educación como práctica de la libertad. Madrid: Siglo XXI; 1989.

33. Corrales D, Galindo A, Escobar MA, Palomo L, Magariño MJ. El debate sobre la organización, las funciones y la eficiencia de enfermería en atención primaria: a propósito de un estudio cualitativo. Aten Primaria 2000;4, 214-219.

34. Sancho Viudes S, Vidal Thomás C, Canellas Pons R, Caldés Pinilla MJ, Corcoll Reixach J, Ramos Montserrat M. Análisis de situación y propuestas de mejora en enfermería en atención primaria de Mallorca: un estudio con grupos focales. Revista Española de Salud Pública 2002;76:531-543..

35. Pérez Jarauta MJ, cols. En la madurez...Guía educativa para promover la salud de las mujeres. Pamplona: Gobierno de Navarra. Departamento de Salud. Instituto de Salud Pública;1996.

36. Pérez Jarauta MJ, cols. Documento básico de Metodología de Educación para la Salud grupal. Pamplona: Gobierno de Navarra. Departamento de Salud. Instituto de Salud Pública;1998.

37. Dirección Provincial de Madrid. Insalud. Recomendaciones metodológicas básicas para elaborar un proyecto educativo. Madrid: Insalud; 1999.

38. Turabián JL, Pérez-Franco B, Hernández I, Arenosa A, Calvo R. Evaluación cuantitativa de un método de educación para diabéticos en consultas grupales. Centro de Salud 1998;6:290-293.

39. Unión Internacional de Promoción de la Salud y Educación para la Salud. La evidencia de la eficacia de la Promoción de la Salud. Parte una y dos. Madrid: Ministerio de Sanidad y Consumo; 2000.

40. Sánchez-Candamio M. Evaluación de programas de salud desde la perspectiva de la metodología cualitativa. Atención Primaria 1999; 24:487-491.

41. Ribot C, Fernández Tenllado MA, García de León D. Investigación cualitativa en Atención Primaria. Una experiencia con entrevistas abiertas. Aten Primaria 2000; 25: 343-348.

42. Martín Zurro A. Nuevas perspectivas en la evaluación de la atención primaria: las metodologías cualitativas editorial.-. Aten Primaria 2000;25: 605.

43. Sarmiento Gallego M, Martínez Ros MT, Sánchez Sánchez F, Sánchez Esteban JM, Messeguer Zaragoza A, Jiménez Bello JJ. Cómo vemos los sanitarios el previsible futuro de nuestro trabajo en los centros de salud. Aten Primaria 1997; 20:311-314..

44. Viñas Maestre M, Castel Simon S. Opiniones y expectativas de los profesionales de atención primaria sobre el trabajo en equipo. Aten Primaria 2000; 26: 309-313. 